\title{
Wetting behaviour of solid and liquid hydrogen films
}

\author{
Jürgen Klier, Christian Weichhard, Paul Leiderer*
}

Fakultät für Physik, Universität Konstanz, Postfach 5560-M-675, 78457 Konstanz, Germany

\section{Abstract}

Adsorbates on strong binding substrates like $\mathrm{H}_{2}$ on Au show triple-point dewetting. To study this phenomenon in detail, we have reduced the van der Waals interaction of the $\mathrm{H}_{2}$ films with the Au surface, by preplating the substrate with $\mathrm{Ne}, \mathrm{Ar}$ or $\mathrm{CH}_{4}$. From a theoretical model, which considers the deformation of the adsorbate by the attractive van der Waals potential and the lattice mismatch due to different lattice constants of adsorbate and substrate, complete wetting is expected when preplating the substrate. Our experiments show, however, that for all combinations of preplated material the equilibrium $\mathrm{H}_{2}$ film thickness is - apart from small differences - the same as without preplating. This indicates that the present picture of the triple-point wetting of $\mathrm{H}_{2}$ is incomplete. (C) 2000 Elsevier Science B.V. All rights reserved.

Keywords: Hydrogen; Van der Waals interaction; Wetting

Physisorbed films of solid molecular hydrogen are of practical importance for various applications [1]. Unfortunately, however, the preparation of films thicker than a few monolayers (ML) is hampered by the fact that solid hydrogen on most substrates exhibits incomplete wetting (i.e. Stranski-Krastanov growth), so that the hydrogen molecules have to be quench-condensed (a nonequilibrium situation) in order to form, say, a $100 \AA$ thick film. Annealing these films leaves big crystallites connected by a very thin film of typically $3 \mathrm{ML}$ on the substrate [2]. On the basis of a theory by Gittes and Schick [3] one should expect that this scenario can be changed to complete wetting of the solid phase under equilibrium conditions, if the interaction with the substrate is modified: on "usual" substrates the interaction is so strong that strain in the adsorbed film prevents complete wetting; reducing this interaction, e.g. by preplating with a proper material, should reduce the strain and lead to complete wetting, thus allowing to grow thick "epitaxial" hydrogen layers. We have investigated this idea by studying the wetting behaviour of solid and liquid $\mathrm{H}_{2}$ films on Au substrates preplated with thin layers of various inert materials.
The experiments were performed on a glass prism covered with a $40 \mathrm{~nm}$ Au film located in a small optical ${ }^{4} \mathrm{He}$ flow cryostat. The temperature could be varied between 6 and $90 \mathrm{~K}$. The thickness of the adsorbed $\mathrm{H}_{2}$ and preplating films was measured by surface plasmon spectroscopy with a resolution of about $0.1 \mathrm{ML}$ [2].

For $\mathrm{H}_{2}$ on bare $\mathrm{Au}$ surfaces the phenomenon of triple-point dewetting (complete wetting in the liquid phase above the triple-point temperature $T_{3}$ and incomplete wetting below) is well established. ${ }^{1}$ In our preplating study we have first used Ne films, adsorbed on the $\mathrm{Au}$ surface under equilibrium conditions, with a thickness of 1 and $2 \mathrm{ML}$. When adsorbing $\mathrm{H}_{2}$ onto such a substrate at a fixed temperature $T>T_{3}$ a thick film grows as one approaches saturated vapour pressure, which is indicative of complete wetting. In contrast, adsorption isotherms below $T_{3}$ reveal incomplete wetting of solid $\mathrm{H}_{2}$ on $\mathrm{Ne} / \mathrm{Au}$, just like for bare Au substrates. Actually, the $\mathrm{H}_{2}$ thickness for the preplated substrate is even somewhat thinner than without Ne preplating, shown in Fig. 1.

In addition, we have also tried $\mathrm{Ar}$ and $\mathrm{CH}_{4}$ as preplating layers. Preplating with $\mathrm{Ar}$ had no influence on the adsorbed $\mathrm{H}_{2}$ thickness within our accuracy, i.e. the equi-

*Corresponding author. Tel.: 07531-88-3793; fax: 07531-883091 .

E-mail address: paul.leiderer@uni-konstanz.de (P. Leiderer) 


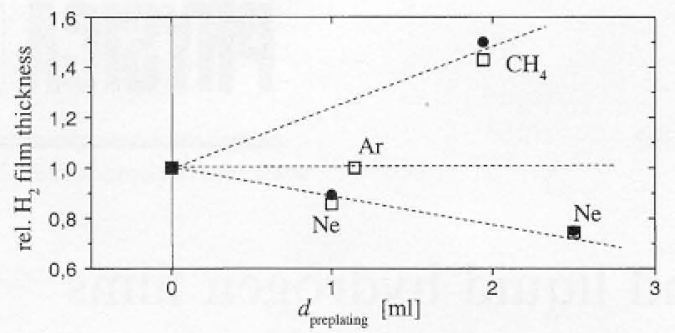

Fig. 1. Maximum $\mathrm{H}_{2}$ film thickness (at saturated vapour pressure) for various preplating layers - with $\mathrm{H}_{2}$ on bare $\mathrm{Au}$ as reference. Dashed lines are just guidance to the eye.

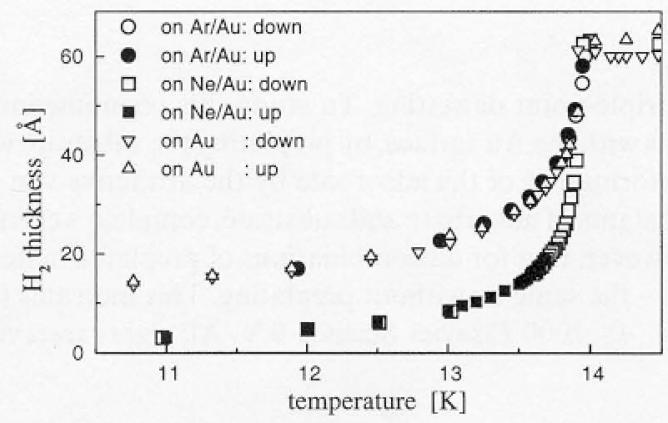

Fig. 2. $T_{3}$-dewetting for pure $\mathrm{H}_{2}$ on $\mathrm{Au}$ and when preplating with $\mathrm{Ne}$ and $\mathrm{Ar}$. $\mathrm{T}_{3}$ of $\mathrm{H}_{2} \approx 14 \mathrm{~K}$.

librium thickness (at saturated vapour pressure) at $T \ll T_{3}$ was $\approx 3 \mathrm{ML}$. Preplating with $2 \mathrm{ML}$ of $\mathrm{CH}_{4}$ increased the equilibrium $\mathrm{H}_{2}$ thickness by a factor of about 1.5 in the solid phase, see Fig. 1. We mention in passing that not only $\mathrm{H}_{2}$, but also our preplating materials $\mathrm{Ne}, \mathrm{Ar}$ and $\mathrm{CH}_{4}$ show $T_{3}$-dewetting on $\mathrm{Au}$, as observed in our measurements. The maximum thickness of the preplating layers was therefore also in this case limited by the equilibrium thickness well below the respective triple-point temperature, again a few ML.
Next to measuring the adsorption isotherms we have also varied the temperature while keeping the system at thermodynamic equilibrium. All these $T$-scans revealed $T_{3}$-dewetting of $\mathrm{H}_{2}$ independent of preplating the $\mathrm{Au}$, see Fig. 2. Below $T_{3}$ the $\mathrm{H}_{2}$ film thickness rapidly decreases to a few ML, quite similar to $\mathrm{H}_{2}$ on bare Au. The absolute thickness depends only weakly on the preplated material in agreement with the data of Fig. 1. Although we observed a slight increase of the $\mathrm{H}_{2}$ thickness when preplating with $\mathrm{CH}_{4}$, it was never possible to change the growth mode from Stranski-Krastanov to Frank-van der Merwe type, as it would have been expected according to Ref. [3]. A similar conclusion has been obtained recently from annealing experiments of quench-condensed solid $\mathrm{D}_{2}$ films on preplated graphite substrates [4]. This leaves the question of the validity of the present picture of the mechanism leading to dewetting below $T_{3}$.

In conclusion both adsorption isotherms and $T$-scans at saturated vapour pressure of $\mathrm{H}_{2}$ have shown $T_{3}$ dewetting of hydrogen, independent of having a bare $\mathrm{Au}$ surface or a preplated one with $\mathrm{Ne}, \mathrm{Ar}$ or $\mathrm{CH}_{4}$. These results are in contrast to present theory and indicate some other dominating influence (e.g. due to inhomogeneities) in such wetting experiments with solid van der Waals films.

\section{Acknowledgement}

This work was supported by the DFG-Schwerpunkt 'Benetzung' under Le 315/20-1.

\section{References}

[1] U. Albrecht et al., J. Low Temp. Phys. 22 (1996) 117.

[2] U. Albrecht et al, Surf. Sci. 283 (1993) 419.

[3] F.T. Gittes, M. Schick, Phys. Rev. B 30 (1984) 209.

[4] L. Fleischmann, Ph.D. Thesis, Mainz, 1998. 